\title{
Mode Sensitivity Exploration of Silica-Titania Waveguide for Refractive Index Sensing Applications
}

\author{
Muhammad A. Butt ${ }^{1, *(1)}$, Andrzej Kaźmierczak ${ }^{1}\left(\mathbb{D}\right.$, Cuma Tyszkiewicz $^{2}{ }^{\oplus}$, Paweł Karasiński ${ }^{2}$ and $^{-}$ \\ Ryszard Piramidowicz ${ }^{1}$ (D) \\ 1 Warsaw University of Technology, Institute of Microelectronics and Optoelectronics, Koszykowa 75, \\ 00-662 Warszawa, Poland; andrzej.kazmierczak@pw.edu.pl (A.K.); ryszard.piramidowicz@pw.edu.pl (R.P.) \\ 2 Silesian University of Technology, Department of Optoelectronics, Krzywoustego 2, 44-100 Gliwice, Poland; \\ cuma.tyszkiewicz@polsl.pl (C.T.); pawel.karasinski@polsl.pl (P.K.) \\ * Correspondence: ali.butt@pw.edu.pl
}

Citation: Butt, M.A.; Kaźmierczak, A.; Tyszkiewicz, C.; Karasiński, P.; Piramidowicz, R. Mode Sensitivity Exploration of Silica-Titania Waveguide for Refractive Index Sensing Applications. Sensors 2021, 21, 7452. https://doi.org/10.3390/ s21227452

Academic Editor: Andrea Cusano

Received: 27 October 2021

Accepted: 6 November 2021

Published: 9 November 2021

Publisher's Note: MDPI stays neutral with regard to jurisdictional claims in published maps and institutional affiliations.

Copyright: (c) 2021 by the authors. Licensee MDPI, Basel, Switzerland. This article is an open access article distributed under the terms and conditions of the Creative Commons Attribution (CC BY) license (https:// creativecommons.org/licenses/by/ $4.0 /)$.

\begin{abstract}
In this paper, a novel and cost-effective photonic platform based on silica-titania material is discussed. The silica-titania thin films were grown utilizing the sol-gel dip-coating method and characterized with the help of the prism-insertion technique. Afterwards, the mode sensitivity analysis of the silica-titania ridge waveguide is investigated via the finite element method. Silicatitania waveguide systems are highly attractive due to their ease of development, low fabrication cost, low propagation losses and operation in both visible and near-infrared wavelength ranges. Finally, a ring resonator (RR) sensor device was modelled for refractive index sensing applications, offering a sensitivity of $230 \mathrm{~nm} / \mathrm{RIU}$, a figure of merit (FOM) of $418.2 \mathrm{RIU}^{-1}$, and Q-factor of 2247.5 at the improved geometric parameters. We believe that the abovementioned integrated photonics platform is highly suitable for high-performance and economically reasonable optical sensing devices.
\end{abstract}

Keywords: silica-titania integrated waveguides; optical ring resonator; refractive index sensor; sol-gel film deposition; waveguide mode sensitivity

\section{Introduction}

Silica, titania, and silica-titania compounds obtained by the sol-gel process have been extensively investigated because of their potential optical functions [1,2]. They have been manipulated to build planar (1D) and ridge (2D) waveguides (hereafter represented as WGs) with excellent optical properties and negligible optical transmission losses for photonic integrated circuits (PICs) [3-5]. The sol-gel methodology is uncomplicated and cheaper [6]. It is capable of coating large areas and does not need high-temperature processing. It permits the development of high-quality thin films with excellent thermal and mechanical stability $[7,8]$.

A suitable refractive index contrast between the WG layer and substrate is essential for WG operation. Light propagates in the high-index thin film, where it is confined by the mechanism of total internal reflection (TIR) [9]. Adjusting the set-off precursors and solvents, as well as the stoichiometric ratio between the silica and titania compounds and the thermal treatment temperature, will regulate the refractive index and thickness of the thin films formed by the sol-gel method. These WGs can be doped with rare-earth elements, laser dyes, and other organic compounds, enabling silica and titania materials created by the sol-gel method to be used in optical amplifiers, laser-active media, and sensing applications $[10,11]$. The sol-gel method is exceedingly effective and does not require expensive high-tech apparatus. By using the sol-gel method, the refractive index of the WG films can be controlled, and the optical losses suffered by these WGs are comparable to WGs acquired from the chemical vapor deposition (CVD)/low-pressure chemical vapor deposition (LPCVD) method [12,13]. In our previous studies [13-15], rib WGs and 
directional couplers (DCs) were fabricated by using traditional optical photolithography and wet chemical etching in $\mathrm{SiO}_{2}: \mathrm{TiO}_{2}$ WG layers.

In this paper, we report advances in the development of a novel WG material platform based on $\mathrm{SiO}_{2}: \mathrm{TiO}_{2}$ material for applications in integrated photonic devices. Moreover, numerical analysis of the mode sensitivity of the $\mathrm{SiO}_{2}: \mathrm{TiO}_{2}$ ridge WG is presented. The work is concluded with a numerical analysis of a simple optical ring resonator (RR)-based sensor device to be fabricated using the presented technology. The conducted simulations of the proposed optical RR sensor structure have proven its potentially high sensitivity comparable with the sensitivity offered by the leading configurations of competitive photonic sensors. For example, when comparing our sensor with a plasmonic sensor based on $\mathrm{Au} / \mathrm{Si}$ nanorods [16], one can state that the sensitivity offered by our device is of the same order of magnitude ( $230 \mathrm{~nm} / \mathrm{RIU}$ in the case of $\mathrm{SiO}_{2}: \mathrm{TiO}_{2} \mathrm{RR}$ vs. $\left.340 \mathrm{~nm} / \mathrm{RIU}\right)$. However, our sensor offers a much better quality factor (2247.5 vs. 10.09); consequently, it should be much easier to identify a central wavelength of the resonance peak and correctly identify the resonance peak shift. Considering the potentially much easier fabrication technology (with the use of direct nanoimprint) and cascading devices (with a serial connection of several RR), the proposed design of the sensor device is a very promising candidate for future implementation in multiparametric lab-on-a-chip sensors.

\section{2. $\mathrm{SiO}_{2}: \mathrm{TiO}_{2}$ Sol-Gel-Derived WG Films}

In our research, we consider the use of low-propagation-loss $\mathrm{SiO}_{2}: \mathrm{TiO}_{2}$ WG layers that are fabricated using sol-gel-derived deposition method with a dip-coating technique. This technology has been mastered at the Silesian University of Technology [6]. The fabrication procedure is depicted schematically in Figure 1, as quite a straightforward method and does not require an expensive setup.

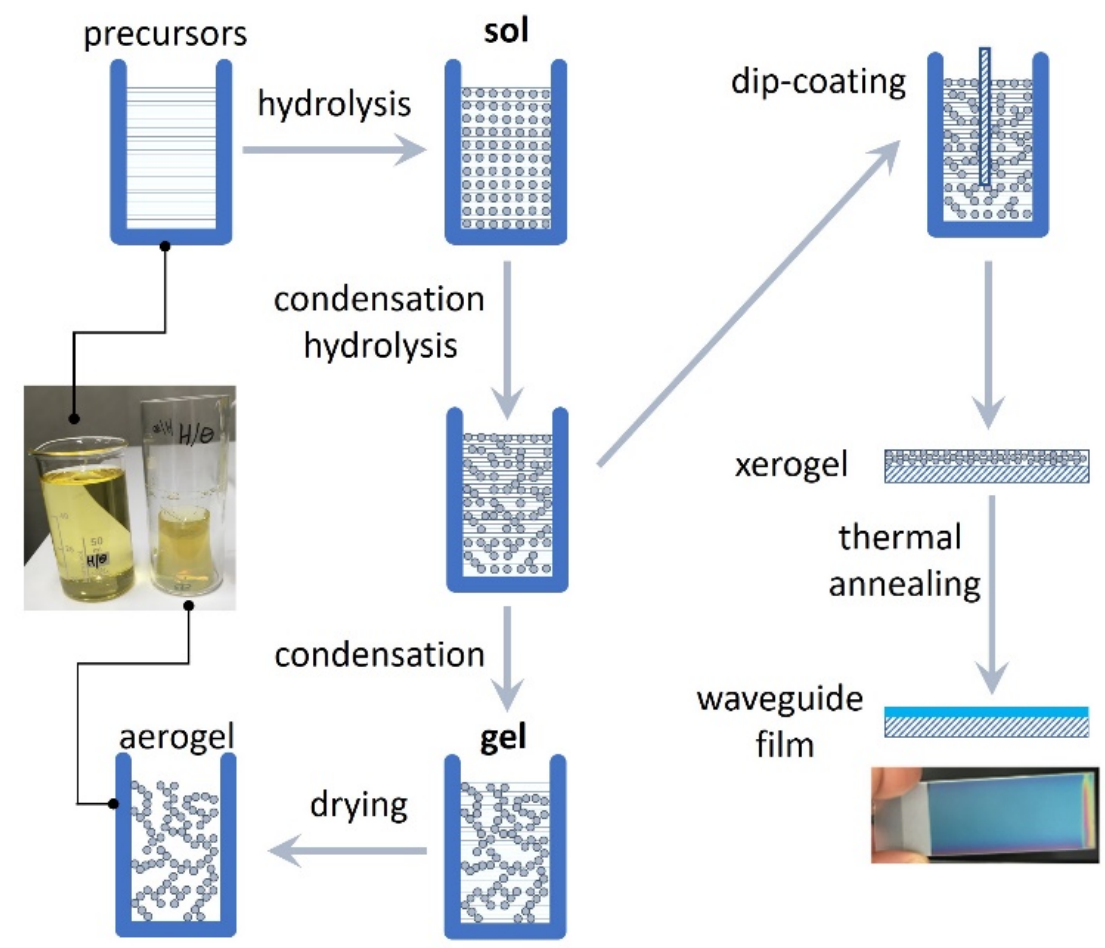

Figure 1. Schematic representation of the $\mathrm{SiO}_{2}: \mathrm{TiO}_{2} \mathrm{WG}$ film deposition process.

In this process, tetraethyl ortotitanate (TET) is used as a titanium dioxide $\left(\mathrm{TiO}_{2}\right)$ precursor, and tetraethyl ortosilicate (TEOS) is used as silicon dioxide $\left(\mathrm{SiO}_{2}\right)$. The two precursors do not dilute in water; therefore, the ethyl alcohol $\left(\mathrm{C}_{2} \mathrm{H}_{5} \mathrm{OH}\right)$ is used as the homogenizing factor. Hydrolysis and condensation reactions are catalyzed with the use of hydrochloric acid. 
A proper rectangular glass substrate is dip-coated in fabricated sol and subsequently annealed by heating it up to a temperature of $400{ }^{\circ} \mathrm{C}$ for $30 \mathrm{~min}$.

This fabrication procedure has several fundamental advantages, including:

- Simplicity and low-cost fabrication because no expensive equipment, such as a PECVD system, is necessary;

- Precise control of WG thickness with proper adjustment of the substrate withdrawal speed;

- Precise control of refractive index with proper adjustment of the stoichiometric ratio between precursor components;

- Low propagation loss thanks to the high smoothness of fabricated WG films;

- Possibility of etch-less fabrication with the implementation of direct nano-imprint lithography (NIL) on non-hardened WG films;

- Scalability with an increment of the number of substrates being dip-coated at the same time.

The first four of the above-mentioned advantages have been demonstrated in our previous research [6]. The possibility of using the NIL technique was partially demonstrated with the development of shallowly patterned vertical grating coupler sensors [17]. The scalability will be investigated in our forthcoming research after the successful development of a fully efficient WG film fabrication and patterning technique when the technology will reach the industrial maturity standard.

The possibility of precisely controlling the $\mathrm{SiO}_{2}: \mathrm{TiO}_{2}$ WG film thickness and the refractive index was investigated by the procurement and characterization of a series of films fabricated with different $\mathrm{SiO}_{2}: \mathrm{TiO}_{2}$ precursor ratios and different substrate withdrawal speeds from the sol. Fabricated WG films were investigated using the optical ellipsometry technique; the obtained results are shown in Figure 2.
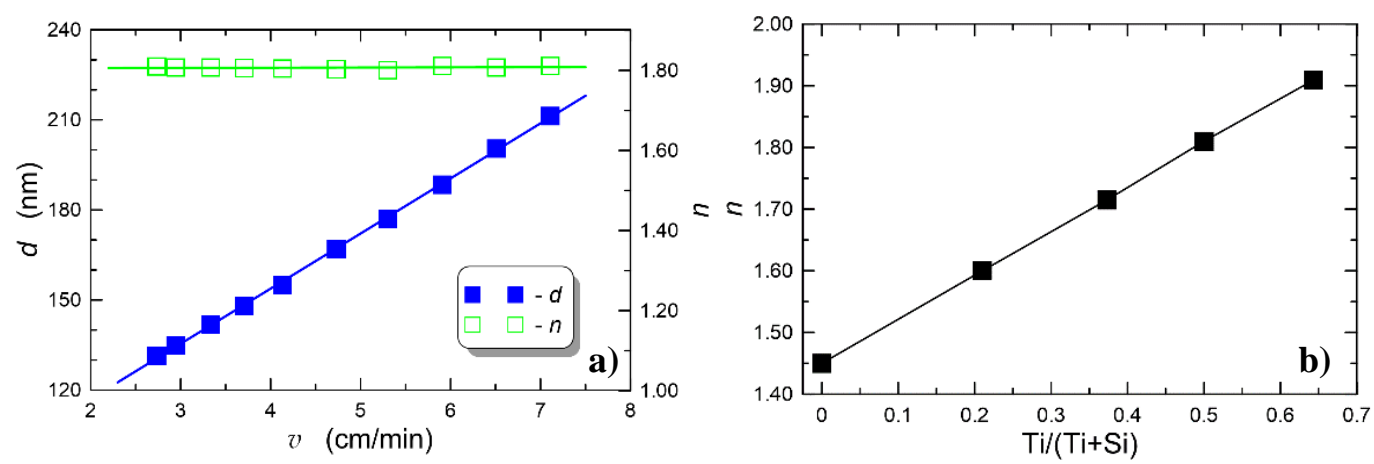

Figure 2. Dependence of WG film thickness and refractive index on withdrawal speed of the substrate from the sol (a), and the dependence of refractive index on the stoichiometric ratio between $\mathrm{Si}$ and $\mathrm{Ti}$ components in the sol (b).

As one can observe in Figure 2a, the thickness of the WG film depends linearly on the withdrawal speed from sol, whereas the refractive index depends on $v$ only to a minor extent. The concentration of titanium in a sol, governed by the relative concentration of respective precursors of silica and titania, has a considerable impact on the refractive index of composite $\mathrm{SiO}_{\mathrm{x}}: \mathrm{TiO}_{\mathrm{y}} \mathrm{WG}$ films. The effect of titanium concentration on the refractive index of such WG films is shown in Figure $2 b$. Square markers corresponding to refractive index values were obtained experimentally. Characteristics presented in Figure 2a were obtained for a composition characterized by the ratio of Ti:Si $=1: 1$.

The surface topology of $\mathrm{SiO}_{2}-\mathrm{TiO}_{2}$ films fabricated on soda-lime glass substrates was investigated using atomic force microscopy (AFM). An exemplary AFM image (left) and two-axis top-surface line scans (right) are shown in Figure 3. 

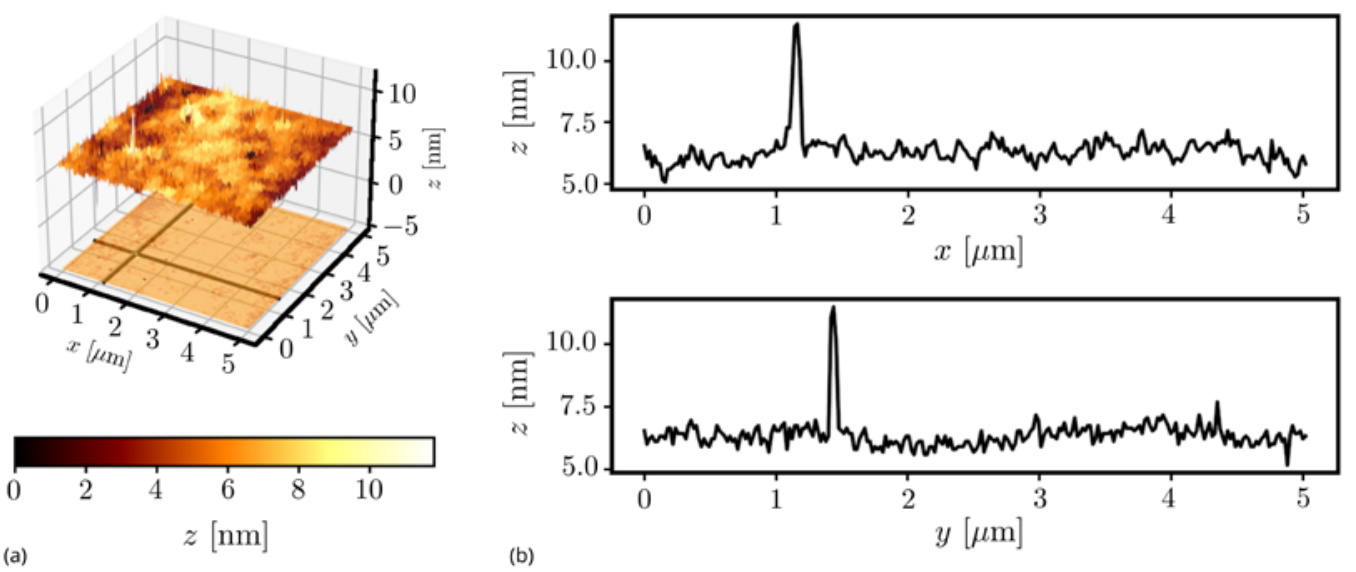

Figure 3. An AFM image (a) and roughness profiles (b) of the top surface of the $\mathrm{SiO}_{2}: \mathrm{TiO}_{2}$ film deposited on a soda-lime glass substrate.

The selected sample was measured in nine different spots using the Ntegra Spectra system. Eight spots were located on the circumference of a square with dimensions of $10 \times 10 \mathrm{~mm}^{2}$. The last, the ninth, was located in the center of that square. There were registered scans of fields having dimensions of $5 \times 5 \mu \mathrm{m}^{2}, 2 \times 2 \mu \mathrm{m}^{2}$ and $1 \times 1 \mu \mathrm{m}^{2}$, three per each size. This enabled determining the dependence of root mean square surface roughness $\sigma_{\text {RMS }}$ and its maximum deviation from the average on scan dimensions, as presented in Figure 4.

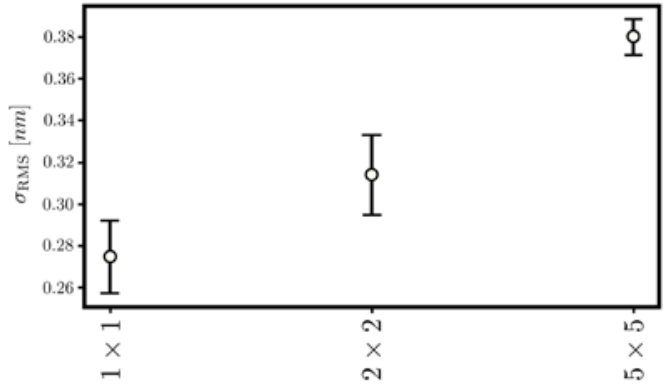

a)

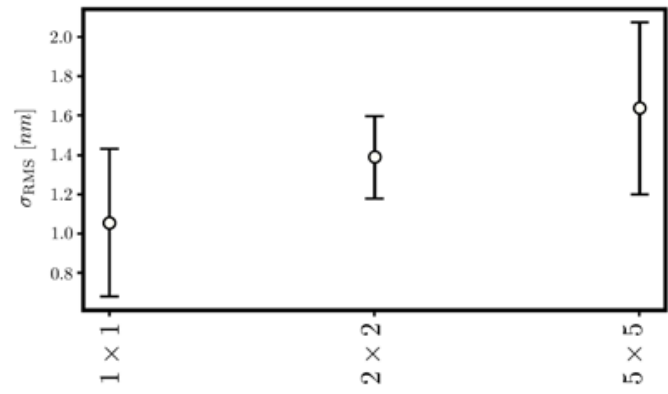

b) scan size $\left[\mu m^{2}\right]$

Figure 4. Variation of the root mean square surface roughness and its maximum deviation from the average concerning scan field dimensions for (a) $\mathrm{SiO}_{2}: \mathrm{TiO}_{2}$ film deposited on a $\mathrm{BK} 7$ glass substrate and (b) a bare substrate.

The surface roughness increases with the length of the probed area, because on larger areas, it is more likely to encounter sharp peaks such as that present in Figure 4a and whose center coordinates are $\mathrm{x}=1.16 \mu \mathrm{m}$ and $\mathrm{y}=1.43 \mu \mathrm{m}$. Nevertheless, the roughness of $\mathrm{SiO}_{2}-$ $\mathrm{TiO}_{2}$ films is very low. It is approximately four times lower than the surface roughness of the BK7 glass substrate (Figure $4 \mathrm{~b}$ ). Additionally, the operation of fabricated WG films was verified by inserting light into the WG slab (using the prism-insertion technique) and observing the stray light propagating in the WG. In Figure 5, two $\mathrm{SiO}_{2}: \mathrm{TiO}_{2}$ WG films excited with light of wavelength $\lambda=677 \mathrm{~nm}$ are shown. Each picture shows a streak of light scattered on WG film interfaces accompanied by the distribution of light intensity $I$ recorded along with the streak. Intensity is expressed in arbitrary units falling within the interval from $I=0$ to $I=255$, because light streaks were registered using a monochromatic Panasonic WV-BL730 camera. Its CCD sensor allows recording images with 8-bit depth. 

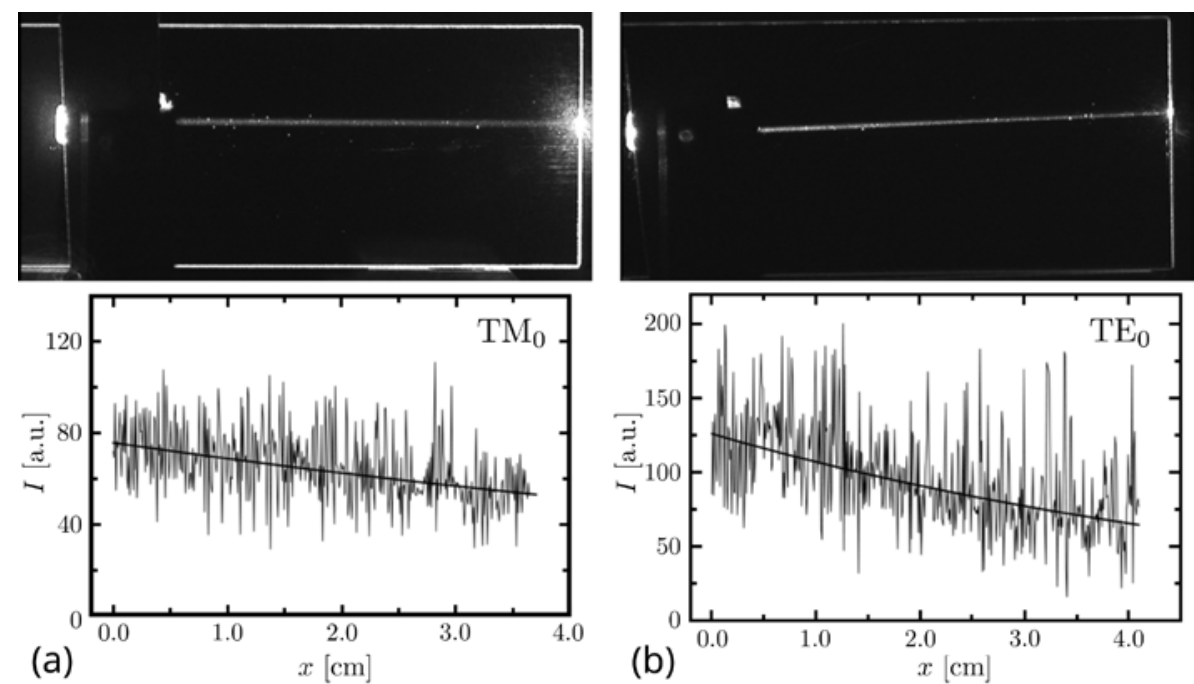

Figure 5. Demonstration of the light propagation in $\mathrm{SiO}_{2}: \mathrm{TiO}_{2} \mathrm{WG}$ films. Light intensity $I$ is given in arbitrary units. A scattering streak of light (top) and its intensity profile (bottom)is shown for the TM (a) and TE (b) polarization.

The experiment demonstrated in Figure 5 clearly shows that the fabricated WG films have very good properties in terms of light propagation. Based on the demonstrated intensity profiles, the value of the propagation loss for these WG films has been estimated to be $\alpha=(0.34 \pm 0.03) \mathrm{dB} / \mathrm{cm}$ for $\mathrm{TM}_{0}$ mode and $\alpha=(0.71 \pm 0.08) \mathrm{dB} / \mathrm{cm}$ for $\mathrm{TE}_{0}$ mode.

With accomplished experimental investigation, it was possible to confirm that the developed WG film fabrication technology allows for full control of the film thickness and refractive index of procured WG films. Furthermore, the fabricated films have very smooth surfaces, resulting in low propagation loss. It is therefore quite fair to state that the developed films are good candidates for further use in the construction of highly integrated sensor structures, the designs of which will be discussed hereafter in forthcoming sections of the paper.

The fabrication procedure for more complex WG structures (including optical RRs) using developed $\mathrm{SiO}_{2}: \mathrm{TiO}_{2}$ WG films are currently being developed in a twofold manner. In a more conventional procedure, the WG film is deposited using a dip-coating technique from the sol and subsequently thermally annealed as shown in Figure 1. Furthermore, the electron beam resist is deposited on the WG film, and e-beam lithography is used to pattern the resist. The WG film will subsequently be dry etched to replicate the pattern from the resist.

In a more innovative (and potentially with less fabrication effort required) procedure, the dip-coating step will be followed by direct nanoimprinting of the RR pattern on nonhardened WG film and subsequent thermal hardening of patterned WG structures. Both solutions are currently under development.

\section{Effective Refractive Index Analysis}

Figure 6 presents a schematic representation of a WG structure that will be investigated hereafter. It is a ridge WG composed of a $\mathrm{SiO}_{2}: \mathrm{TiO}_{2}$ core deposited on a standard glass substrate. The width and height of the WG core are denoted as $W$ and $H$, respectively. The refractive indexes of the core and substrate were fixed at 1.8 (see Figure 2) and 1.5135 [18], respectively, which corresponded to the refractive index of fabricated WG films. The WG was placed in an aqueous medium of refractive index $=1.33$. The numerical analysis was conducted via a 2D finite element method (2D-FEM) in COMSOL Multiphysics software. The entire device structure was divided into triangular elements with a predefined "Extremely fine" mesh grid size. The effective refractive index of the propagating mode was calculated for a WG of different heights and utilized to solve the problem. Scattering boundary conditions (SBCs) were applied on the outer boundary of the 
WG geometry to make the boundary transparent for the scattered electromagnetic waves. The sensing capability of the abovementioned platform was analyzed by modelling the RR structure for refractive index sensing applications.

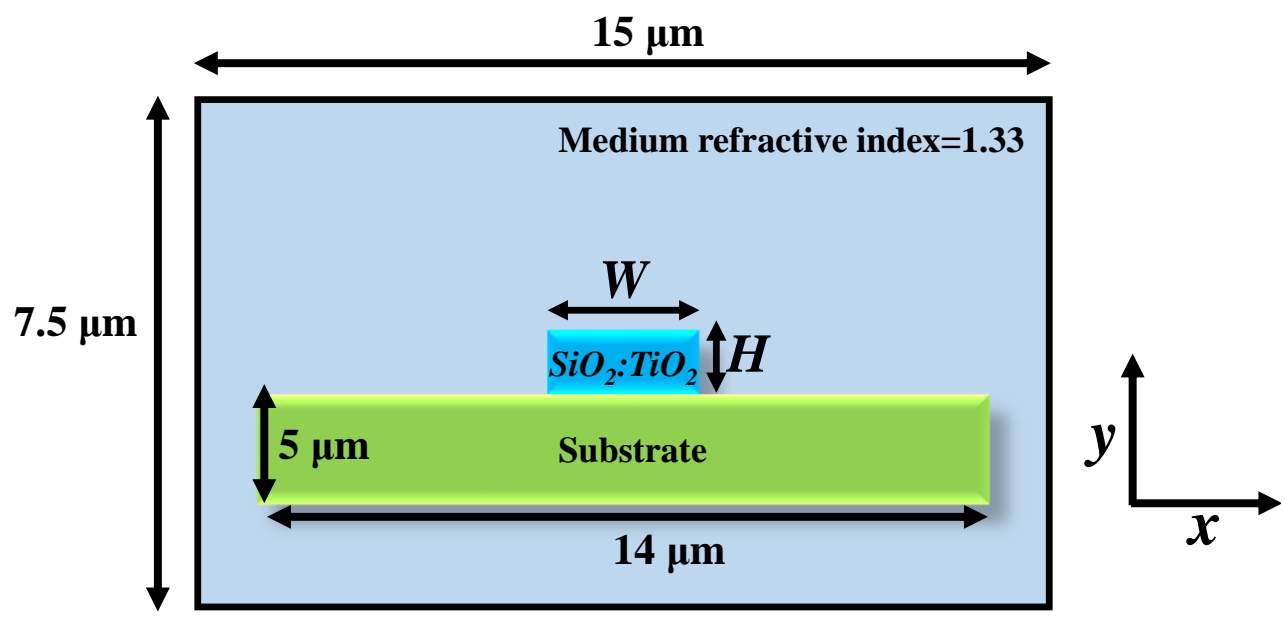

Figure 6. $\mathrm{SiO}_{2}: \mathrm{TiO}_{2}$ ridge $\mathrm{WG}$ scheme.

First, the real part of the effective refractive index $\left(n_{\text {eff }}\right)$ of the propagating mode was calculated for different geometric parameters of the WG core. As shown in the previous section, in our lab, we can deposit a smooth, thin layer of $\mathrm{SiO}_{2}: \mathrm{TiO}_{2}$ on glass with the help of the sol-gel dip-coating method $[6,19]$. Furthermore, the WG film thickness can be precisely adjusted with the properly selected withdrawal speed of the substrate from sol. In [20], a linear dependence between the withdrawal speed of the sample and the thickness of the deposited $\mathrm{SiO}_{2}: \mathrm{TiO}_{2}$ film has been shown, with the film thickness ranging from $200 \mathrm{~nm}$ to $350 \mathrm{~nm}$. Recently, with further technological development, we have been able to demonstrate WG films with thicknesses reaching $500 \mathrm{~nm}$. Consequently, the mode behavior has been analyzed at different core dimensions.

Figure $7 \mathrm{a}-\mathrm{d}$ present the $\operatorname{Re}\left(n_{\text {eff }}\right)$ of the TE-mode at $677 \mathrm{~nm}$. The $n_{\text {eff }}$ of the mode increases as $H$ and $W$ of the WG core increases, which leads to the strong confinement of light in the core. This attribute is beneficial for designing optical interconnects where low transmission losses are desirable. However, for sensing applications, slightly leaky modes are favorable because they can provide enhanced sensitivity due to the strong light-matter interaction [21]. The mode propagating in the WG of $H=200 \mathrm{~nm}$ is highly sensitive to the ambient medium as compared to the mode propagating in the WG core of $H=500 \mathrm{~nm}$. This is because a large portion of the evanescent field is propagating on the surface of the WG core. When the ambient refractive index increases from 1.33 to 1.39 , a shift in the $n_{\text {eff }}$ is observed, with the rate of shift dependent on the $H$ of the WG core. 

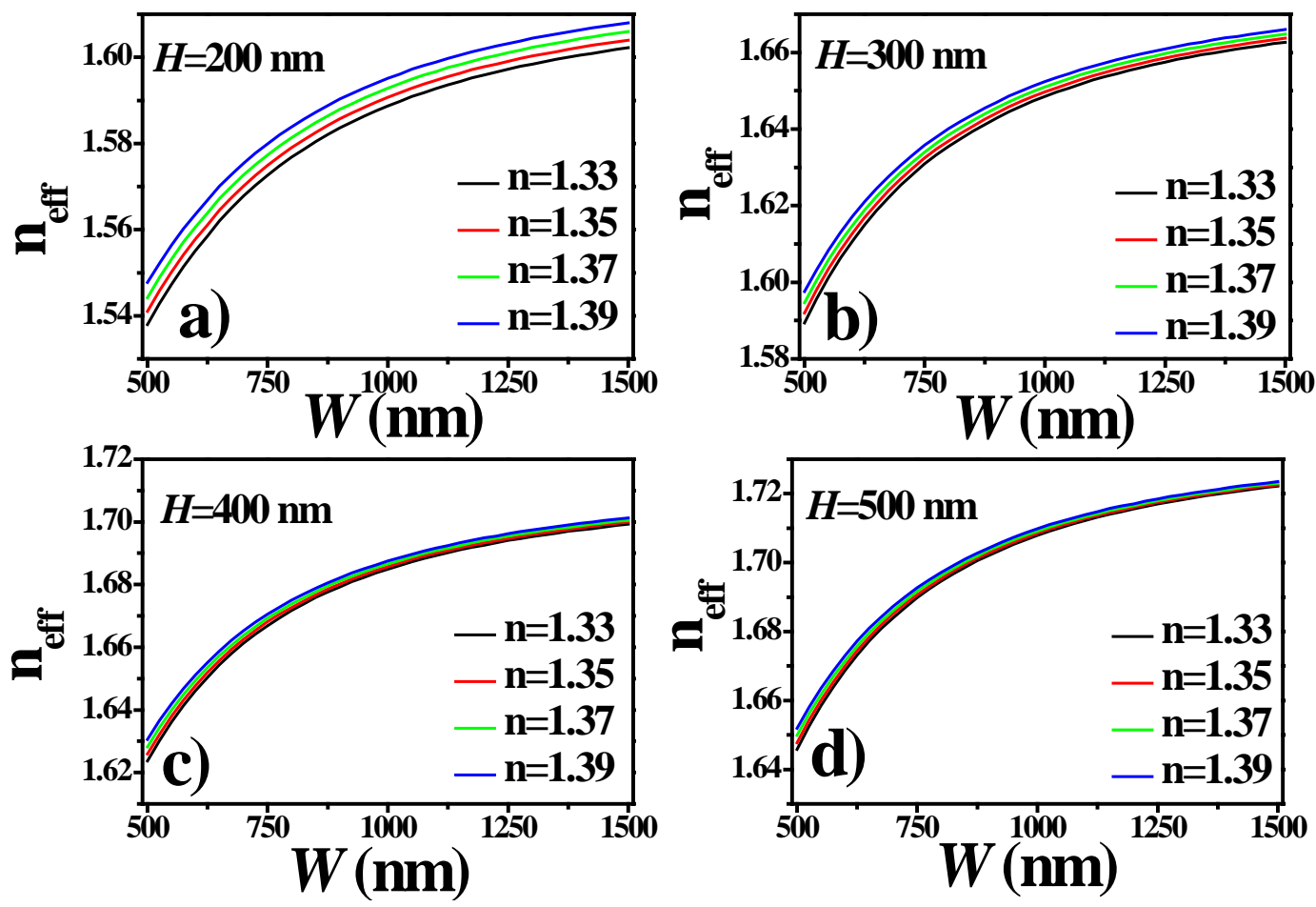

Figure 7. $\operatorname{Re}\left(n_{\text {eff }}\right)$ versus $\mathrm{W}$ for the $\mathrm{H}$ of (a) $200 \mathrm{~nm}$, (b) $300 \mathrm{~nm}$, (c) $400 \mathrm{~nm}$ and (d) $500 \mathrm{~nm}$. Please note that only the TE-fundamental mode was considered.

For optical communication or sensing applications, single-mode WG operation is appropriate. Therefore, $n_{\text {eff }}$ calculation for WG geometry is an initial step to designing WGs of any type. In Figure 8, the normalized E-field distribution of the TE-polarized mode is plotted. For $W=500 \mathrm{~nm}$, the WG supports a fundamental mode for $H=200 \mathrm{~nm}, 300 \mathrm{~nm}$, $400 \mathrm{~nm}$ and $500 \mathrm{~nm}$. However, as $W$ approaches $1000 \mathrm{~nm}$ and beyond, the WG geometry starts supporting higher-order modes $\left(\mathrm{TE}_{10}\right.$ and $\left.\mathrm{T}_{20}\right)$. Therefore, special attention should be given while designing optical devices where single-mode operation is desirable.
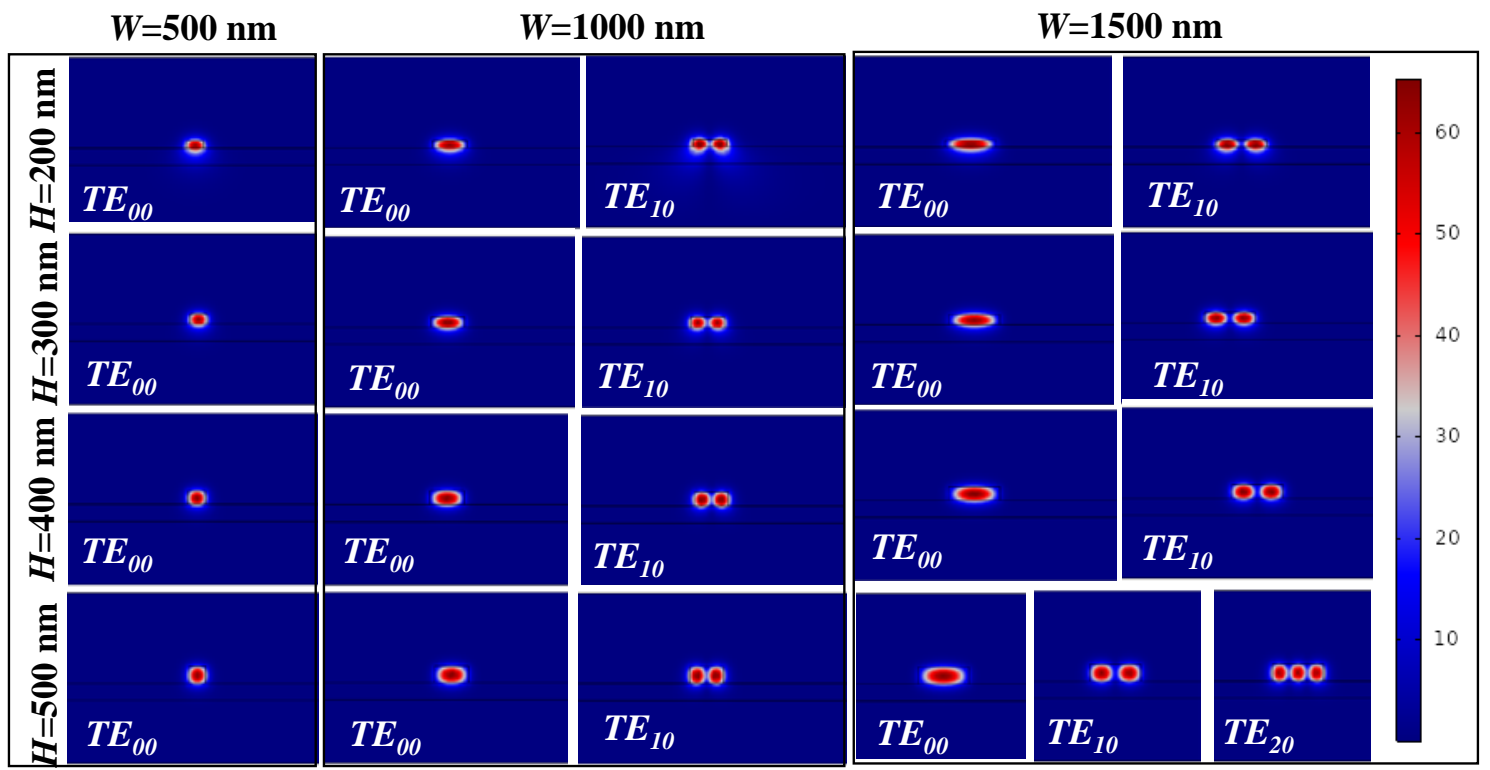

Figure 8. TE-mode distribution in ridge WG with different geometric parameters at an operational wavelength of $677 \mathrm{~nm}$. 


\section{Mode Sensitivity Analysis}

The mode sensitivity analysis of a WG structure is vital. Based on this information, suitable WG dimensions are selected for sensing applications [22,23]. It can be determined by utilizing the following expression:

$$
S_{\text {mode }}=\frac{\Delta n_{e f f}}{\Delta n},
$$

where $\Delta n_{\text {eff }}$ and $\Delta n$ are the change in the effective refractive index and change in ambient refractive index, respectively. From Figure 9, it can be seen that the propagating mode is highly responsive to the ambient medium at small values of $H$ and $W$. For instance, a mode sensitivity of $0.162 \Delta n_{\text {eff }} / \Delta n$ was acquired for the WG geometry of $W=500 \mathrm{~nm}$ and $H=200 \mathrm{~nm}$ for $\Delta n=0.06$. On the other hand, the sensitivity of the WG reduced to 0.097 $\Delta n_{\text {eff }} / \Delta n$ at $W=1500 \mathrm{~nm}$ and $H=200 \mathrm{~nm}$. Further reductions in sensitivity were noticeable as $H$ increased to $500 \mathrm{~nm}$. However, the highly sensitive devices produced a drawback of high propagation loss of the mode. Therefore, there is always a compromise between high sensitivity and high optical losses.

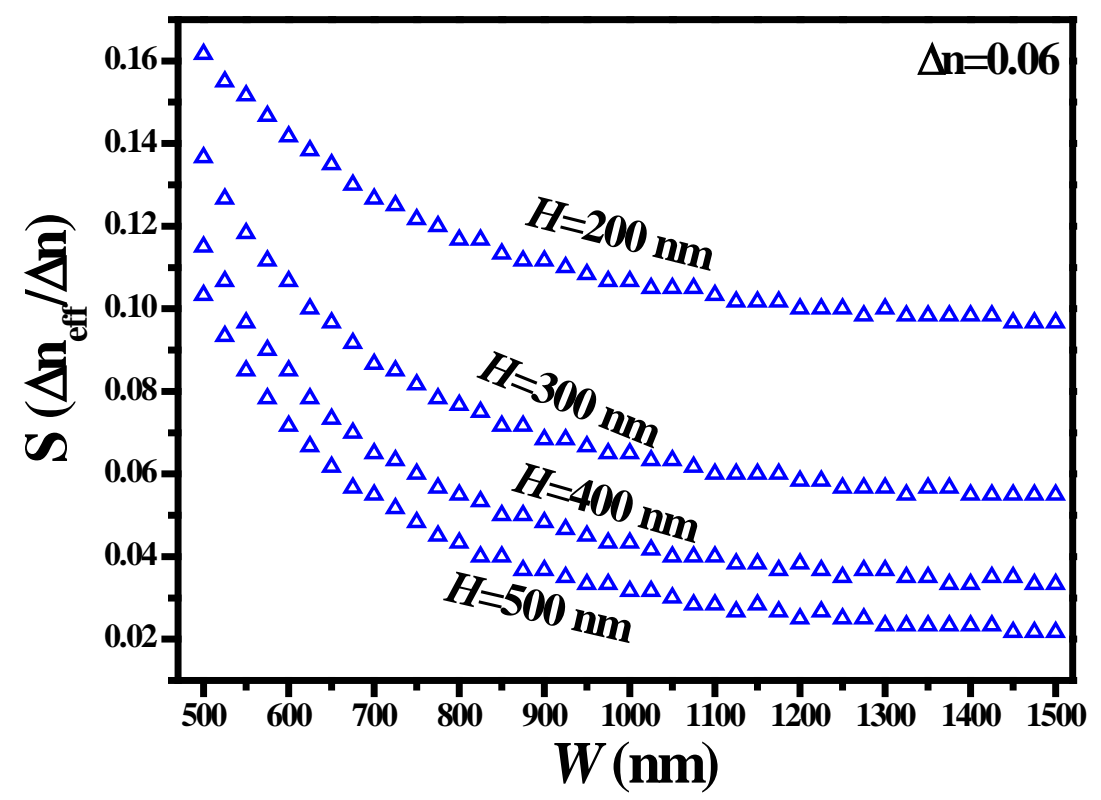

Figure 9. Mode sensitivity analysis of a ridge WG.

\section{Ring Resonator Sensor Based on a Silica-Titania Platform}

Furthermore, an RR device was modelled to numerically investigate the refractive index sensing capabilities of the $\mathrm{SiO}_{2}: \mathrm{TiO}_{2}$ optical WG. Fabrication of the proposed RR structure is currently in progress. There are three vital parameters, sensitivity $(S)$, figure of merit (FOM) and $Q$-factor, which should be well-thought-out while designing the sensing devices $[24,25]$. The sensitivity of the $R R$ is calculated by using the following expression [26]:

$$
S=\frac{\Delta \lambda_{\text {res }}}{\Delta n},
$$

where $\Delta \lambda_{\text {res }}$ and $\Delta n$ are the change in resonance wavelength and ambient refractive index, respectively. FOM is the ratio between the sensitivity and full width at half maximum (FWHM) of the resonance dip, which is expressed as:

$$
F O M=\frac{S}{F W H M},
$$


Integrated resonators with high $Q$-factors are principally required for a wide range of applications, for instance, narrow-bandwidth filters, high-efficiency non-linear optic devices, high-performance lasers and high-sensitivity sensors [27-30]. The Q-factor of the proposed device can be calculated as:

$$
Q-\text { factor }=\frac{\lambda_{\text {res }}}{F W H M},
$$

The RRs are highly responsive to changes in the ambient medium, which result in the redshift of the resonance wavelength [31]. The amount of shift in the resonance wavelength is dependent on the geometric parameters of the device. The schematic of the RR is shown in Figure 10, where $W_{b u s}$ and $W_{\text {ring }}$ represent the width of the bus WG and ring WG, respectively.

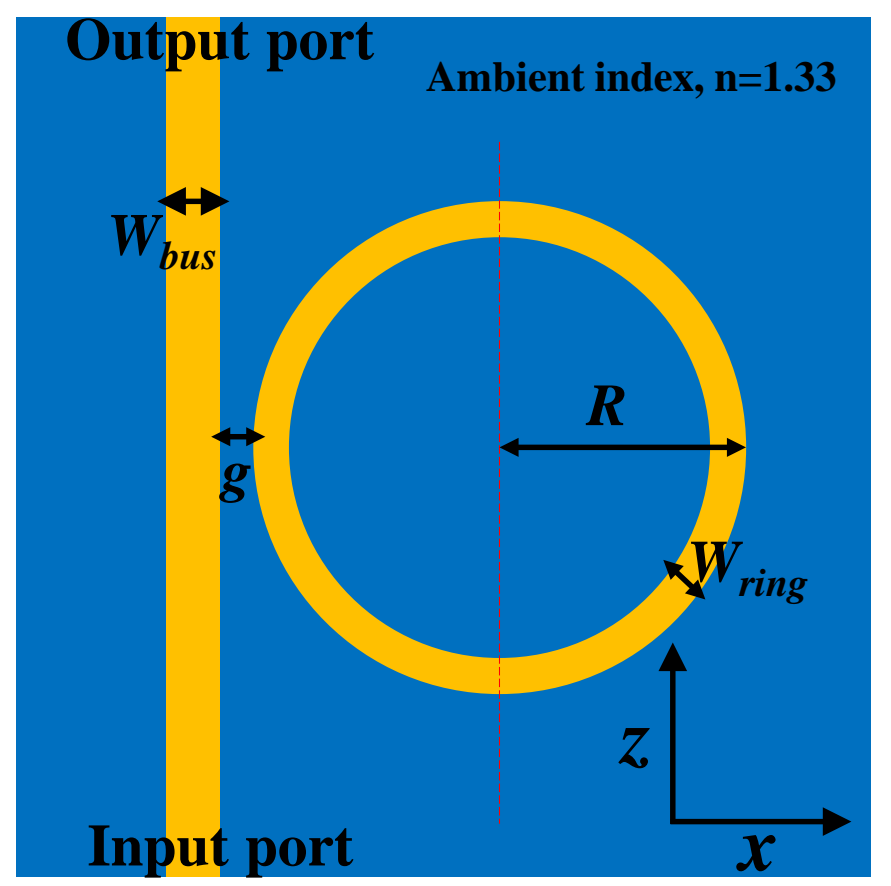

Figure 10. Schematic representation of an $\mathrm{RR}$ based on $\mathrm{SiO}_{2}: \mathrm{TiO}_{2}$ optical WG.

The radius of the ring (denoted as $R$ ) was chosen to be $15 \mu \mathrm{m}$ based on the computational limit of our computer. The coupling distance between the bus WG and the ring is denoted as $g$. The ambient medium was filled with a dielectric material of $n=1.33$. From a practical point of view, the height $(H)$ of the device design was fixed at $200 \mathrm{~nm}$, which could be obtained in a single dip-coating process, and the $n_{\text {eff }}$ values of the $\mathrm{Si}$ WG were obtained from Figure 7. A TE-polarized plane wave was coupled at the input port of the bus WG, and the output power was collected at the other end. To determine the free spectral range (FSR) of the RR structure, the extinction ratio $(E R)$ was calculated by using the following expression:

$$
E R(\mathrm{~dB})=10 \times \log \left(\frac{P_{\text {out }}}{P_{\text {in }}}\right),
$$

where $P_{\text {out }}$ and $P_{\text {in }}$ are the power at the input and output port of the bus WG, respectively. FSR was calculated in the near-IR wavelength range of $960 \mathrm{~nm}$ to $1000 \mathrm{~nm}$ by utilizing a "parametric sweep function" with a step size of $0.05 \mathrm{~nm}$. This wavelength range is suitable for the ring structure of $H=200 \mathrm{~nm}$; otherwise, in the high-wavelength range, the WG becomes leaky, and light penetrates the substrate. For Figure 11a-c, the geometric parameters of the ring structure used for the analysis are as follows: $R=15 \mu \mathrm{m}, g=100 \mathrm{~nm}$, $W_{\text {bus }}=W_{\text {ring }}=800 \mathrm{~nm}$ and $n=1.33$. The transmission spectrum of the RR is plotted in 
Figure 11a. It is well noting that six resonance dips $\left(\lambda_{\text {dip }}\right)$ were obtained with an FSR of $\sim 6.4 \mathrm{~nm}$ and the FWHM of $\lambda_{\text {dip }}$ was $\sim 0.44 \mathrm{~nm}$, as shown in Figure $11 \mathrm{~b}$.
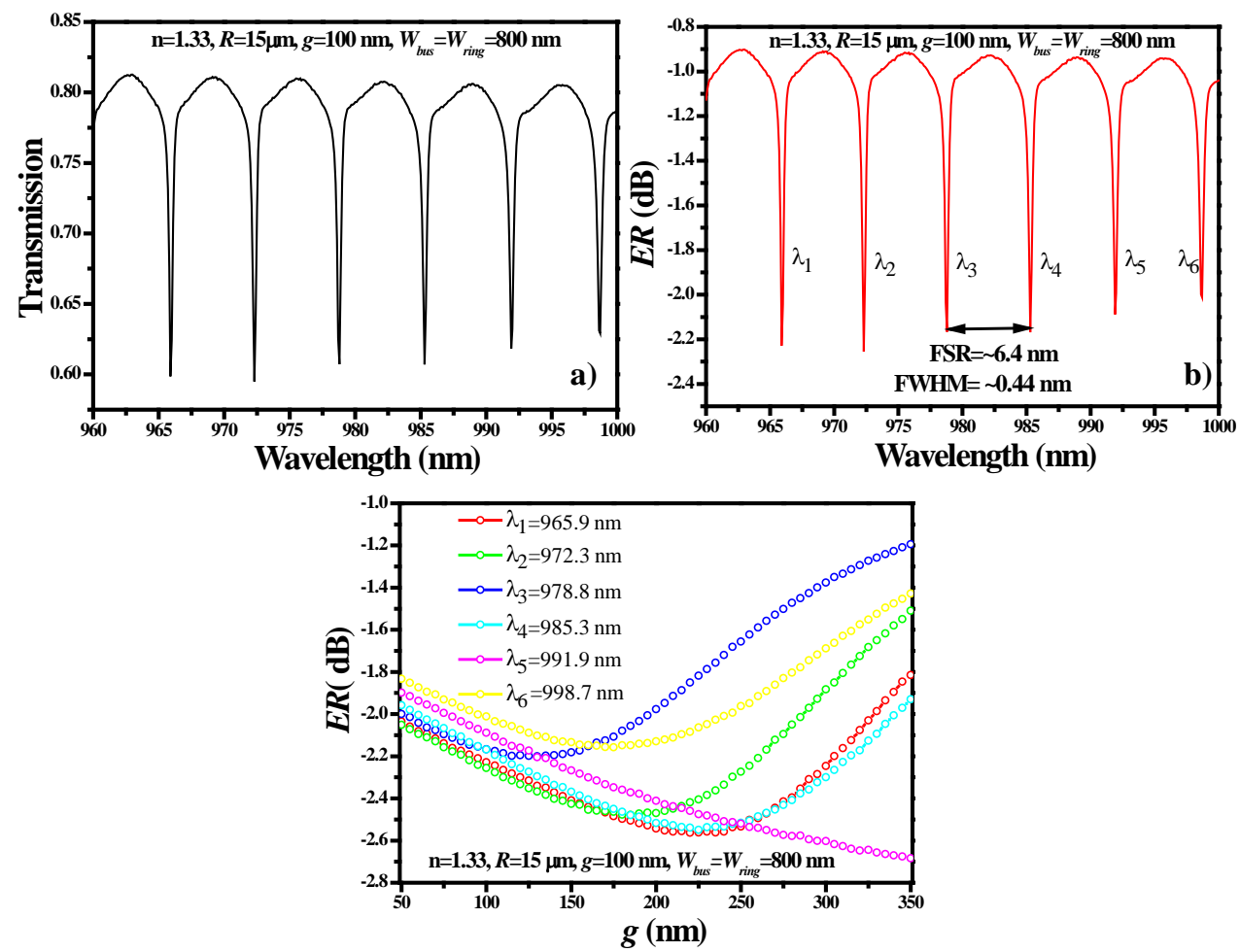

Figure 11. Spectral characteristics of the RR structure: (a) transmission spectrum, (b) FSR versus ER, (c) optimization of $g$. Geometric parameters such as $R=15 \mu \mathrm{m}, g=100 \mathrm{~nm}, W_{b u s}=W_{\text {ring }}=800 \mathrm{~nm}$ and $n=1.33$ are used.

For each $\lambda_{\text {dip }}$, the coupling efficiency (CE) of the light from the bus WG to ring structure was determined as shown in Figure 11c. CE can be determined by the following two methods: (1) integrating the ring structure to calculate the energy stored in it at resonance wavelength; and (2) calculating the $E R$ of the bus WG. Both methods are feasible; however, we have selected the second method and obtained the $E R$ for $g$ in the range of $50 \mathrm{~nm}$ to $350 \mathrm{~nm}$. The maximum $E R$ of $\sim 2.5-2.6 \mathrm{~dB}$ was obtained for $g=250 \mathrm{~nm}$ to $300 \mathrm{~nm}$. In the sensing analysis section, we fixed $g=225 \mathrm{~nm}$, which is one of the optimized coupling gaps to obtain high $E R$.

The sensing capabilities of the proposed sensor design were evaluated by varying the ambient refractive index from 1.33 to 1.365 with a step size of 0.005 . The transmission spectrum (dB) of the RR is plotted for a wavelength range of $980 \mathrm{~nm}$ to $990 \mathrm{~nm}$ where a $\lambda_{4}$ dip is present. The $\lambda_{\text {dip }}$ performed a redshift as the refractive index of the ambient medium increased, as shown in Figure 12a. For this analysis, $W_{b u s}=W_{\text {ring }}=800 \mathrm{~nm}$ was fixed, which provides strong confinement of light in the ring structure which results in a weak evanescent field. Consequently, light-matter interaction is low, resulting in $S=\sim 90 \mathrm{~nm} / \mathrm{RIU}$, $F O M=\sim 204.5 \mathrm{RIU}^{-1}$ and $Q$-factor $=2239$. Normalized E-field distributions at $\lambda=985.3 \mathrm{~nm}$ and $\lambda=987 \mathrm{~nm}$ are plotted in Figure 12b,c, which represent the on-resonance state and off-resonance state, respectively. 


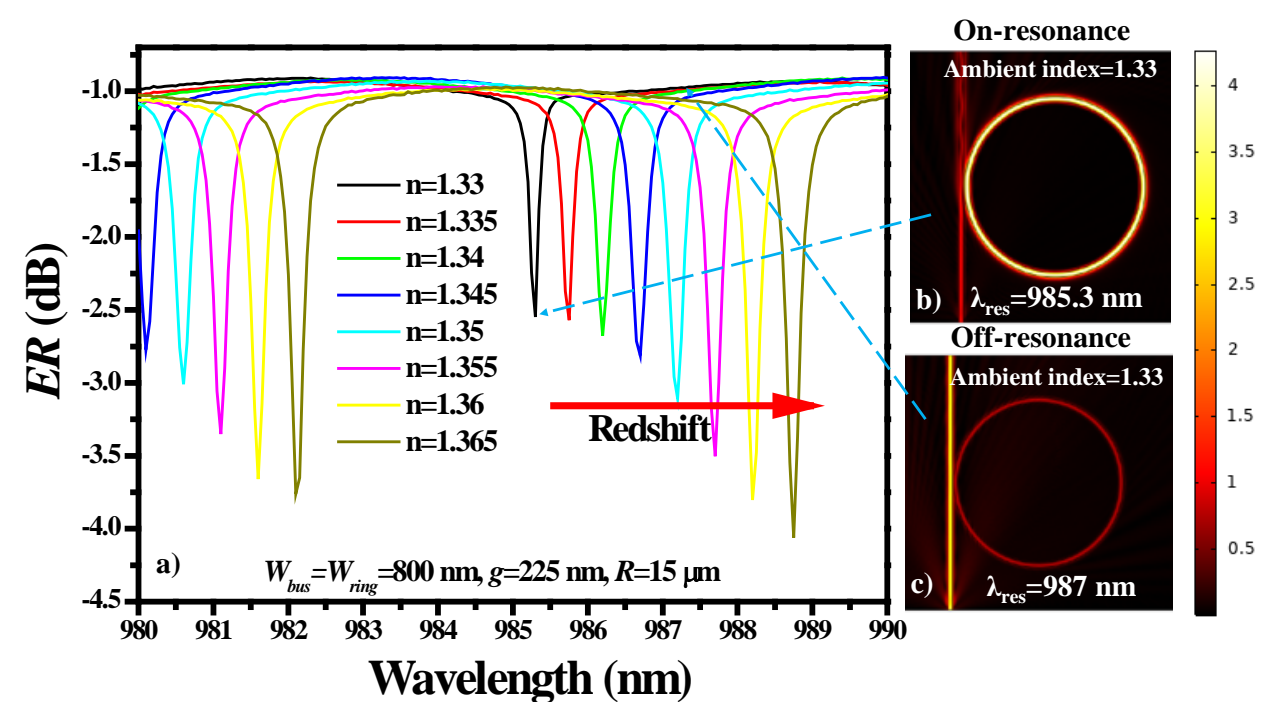

Figure 12. (a) Transmission spectrum of the RR structure in the presence of different ambient refractive indices. Normalized E-field distribution in the RR structure in the (b) on-resonance state and (c) off-resonance state. The geometric parameters of the structure are as follows: $R=15 \mu \mathrm{m}$, $g=225 \mathrm{~nm}$, and $W_{b u s}=W_{\text {ring }}=800 \mathrm{~nm}$.

As mentioned earlier, sensitivity can be enhanced by increasing the light-matter interaction, i.e., increasing the evanescent field ratio (EFR). EFR is defined as the ratio of $\mathrm{E}$ field power in the upper cladding and the total power of the WG mode $[32,33]$. Higher EFR can be obtained by decreasing the width of the ring WG $\left(W_{\text {ring }}\right)$. The device performance is calculated by reducing $W_{\text {ring }}$ from $800 \mathrm{~nm}$ to $500 \mathrm{~nm}$ with a step size of $100 \mathrm{~nm}$. The $\lambda_{\text {res }}$ at $W_{\text {ring }}=500 \mathrm{~nm}$ is $988.9 \mathrm{~nm}$ with an FWHM of $0.44 \mathrm{~nm}$. The remaining parameters such as $W_{\text {bus }}, g$ and $R$ were maintained at $800 \mathrm{~nm}, 225 \mathrm{~nm}$ and $15 \mu \mathrm{m}$, respectively. The $S, F O M$ and $Q$-factor of the proposed device were enhanced to $\sim 230 \mathrm{~nm} / \mathrm{RIU}$ and $\sim 418.2 \mathrm{RIU}^{-1}$ and 2247.5 , respectively, by reducing $W_{\text {ring }}=500 \mathrm{~nm}$, as shown in Figure 13 . Table 1 summarizes the device performance at different values of $W_{\text {ring }}$.

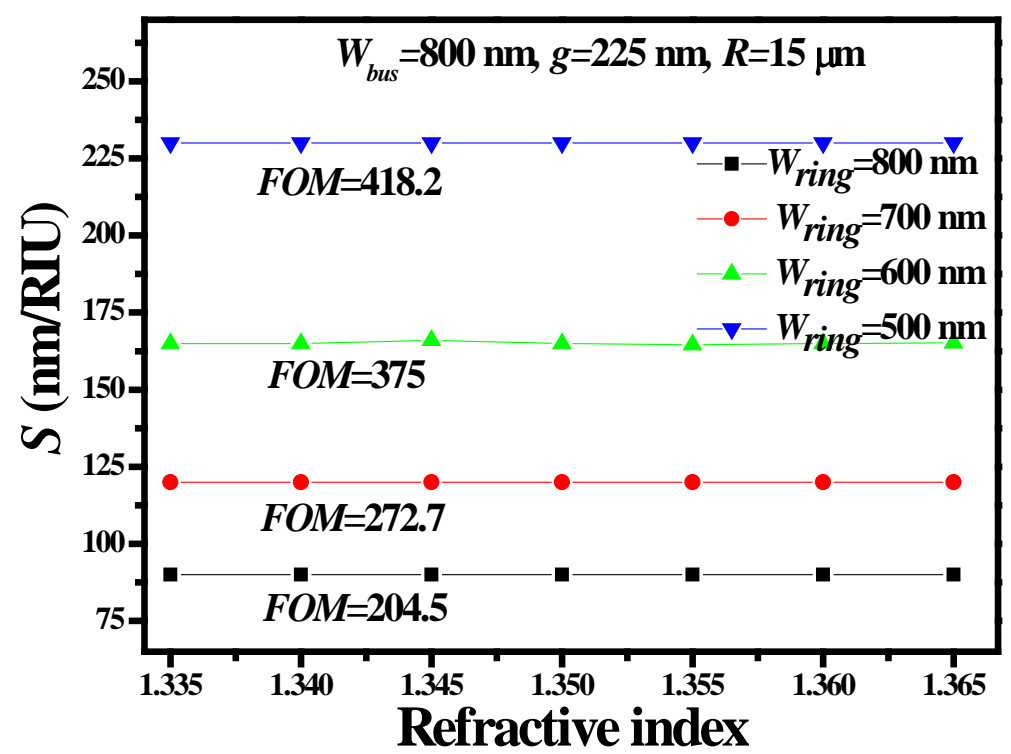

Figure 13. Evaluation of $S$ and FOM based on $W_{\text {ring. }}$. The remaining geometric parameters such as $W_{b u s}, R$ and $g$ were fixed at $800 \mathrm{~nm}, 15 \mu \mathrm{m}$ and $225 \mathrm{~nm}$, respectively. 
Table 1. RR performance for different values of $W_{\text {ring }}$.

\begin{tabular}{cccc}
\hline$W_{\text {ring }}(\mathbf{n m})$ & $S(\mathbf{n m} / \mathbf{R I U})$ & FOM $\left(\mathbf{R I U}^{-\mathbf{1}}\right)$ & $Q_{\text {-Factor }}$ \\
\hline 500 & $\sim 230$ & $\sim 418.2$ & 2247.5 \\
\hline 600 & $\sim 165$ & $\sim 375$ & 2240 \\
\hline 700 & $\sim 120$ & $\sim 272.7$ & 2227.5 \\
\hline 800 & $\sim 90$ & $\sim 204.5$ & 2239 \\
\hline
\end{tabular}

The results presented in this work are highly attractive and comparable to the wellknown silicon photonics and optical fiber-based sensors. For instance, a semiconductor nanowire refractive index sensor for biosensing application was verified, offering $S=235 \mathrm{~nm} / \mathrm{RIU}$ [34]. A long-period fiber grating sensor has been proposed for refractive index sensing applications [35]. The obtained sensitivity value is comparable with silicon nitride slot WG RRs sensors, as shown by Carlborg et al., with an experimental sensitivity of $246 \mathrm{~nm} / \mathrm{RIU}$ with a much more complex RR layout [36]. However, by adjusting the WG thickness, $S_{\text {bulk }}=270 \mathrm{~nm} /$ RIU was demonstrated [37].

\section{Conclusions}

In conclusion, a highly attractive $\mathrm{WG}$ platform based on $\mathrm{SiO}_{2}: \mathrm{TiO}_{2}$ is presented, which can be easily developed at low costs. The wide transparency range of the material makes it an ideal candidate to be employed in the visible and near-IR wavelength range. A mode sensitivity analysis of the WG demonstrates the practical applicability of this system in the sensing field. In the end, an RR device has been proposed for refractive index sensing applications and offers sensitivity in the range of $90 \mathrm{~nm} / \mathrm{RIU}$ to $230 \mathrm{~nm} / \mathrm{RIU}$ based on the geometric parameters of the WG. We believe that this $\mathrm{SiO}_{2}: \mathrm{TiO}_{2}$ platform can find several applications in the implementation of active and passive optical components.

Author Contributions: Conceptualization, M.A.B., A.K., C.T., P.K. and R.P.; methodology, M.A.B., A.K., C.T., P.K. and R.P.; software, M.A.B., A.K., C.T., P.K. and R.P.; validation, M.A.B., A.K., C.T., P.K. and R.P.; formal analysis, M.A.B., A.K., C.T., P.K. and R.P.; investigation, M.A.B., A.K., C.T., P.K. and R.P.; resources, M.A.B., A.K., C.T., P.K. and R.P.; data curation, M.A.B., A.K., C.T., P.K. and R.P.; writing—original draft preparation, M.A.B., A.K., C.T., P.K. and R.P.; writing-review and editing, M.A.B., A.K., C.T., P.K. and R.P.; visualization, M.A.B., A.K., C.T., P.K. and R.P.; supervision, M.A.B., A.K., C.T., P.K. and R.P.; project administration, M.A.B., A.K., C.T., P.K. and R.P.; funding acquisition, M.A.B., A.K., C.T., P.K. and R.P. All authors have read and agreed to the published version of the manuscript.

Funding: The work was partially funded with the project "Hybrid sensor platforms of integrated photonic systems based on ceramic and polymer materials" carried out within the TEAM-NET program of the Foundation for Polish Science financed by the European Union under the European Regional Development Fund, POIR.04.04.00-00-14D6/18-01, P.K. and C.T. acknowledge support of the National Science Center within the project OPUS 13 decision DEC-2017/25/B/ST7/02232. Financial support of COE'2020 conference within the framework of the Excellent Science (Doskonała Nauka, DNK/SP/462114/2020) project of Polish Ministry of Education and Science, MEN is acknowledged.

Institutional Review Board Statement: Not applicable.

Informed Consent Statement: Not applicable.

Acknowledgments: We acknowledge the equal contribution of all the authors.

Conflicts of Interest: The authors declare no conflict of interest.

\section{References}

1. Morosanova, E.I. Silica and silica-titania sol-gel materials: Synthesis and analytical application. Talanta 2012, 102, 114-122. [CrossRef] [PubMed]

2. Lenza, R.F.S.; Vasconcelos, W.L. Synthesis of Titania-Silica Materials by Sol-Gel. Mater. Res. 2002, 5, 497-502. [CrossRef]

3. Karasiński, P.; Jaglarz, J.; Mazur, J. Low loss silica-titania waveguide films. Photonics Lett. Pol. 2010, 2, 1. [CrossRef] 
4. Kazmierczak, A.; Slowikowski, M.; Pavlov, K.; Filipiak, M.; Vervaeke, M.; Tyszkiewicz, C.; Ottevaere, H.; Piramidowicz, R.; Karasinski, P. Efficient, low-cost optical coupling mechnism for $\mathrm{TiO}_{2}-\mathrm{SiO}_{2}$ sol-gel derived slab waveguide surface grating coupler sensors. Opt. Appl. 2020, 4, 539-549.

5. Innocenzi, P.; Martucci, A.; Guglielmi, M.; Armelao, L.; Pelli, S.; Righini, G.; Battaglin, G. Optical and surface properties of inorganic and hybrid organic-inorganic silica-titania sol-gel planar waveguides. J. Non-Cryst. Solids 1999, 259, 182-190. [CrossRef]

6. Karasinski, P.; Tyszkiewicz, C.; Piramidowicz, R.; Kazmierczak, A. Development of integrated photonics based on $\mathrm{SiO}_{2}: \mathrm{TiO}_{2}$ sol-gel derived waveguide layers:state of the art, perspectives, prospective applications. In Proceedings of the SPIE 11364, Integrated Photonics Platforms: Fundamental Research, Manufacturing and Applications, SPIE Photonics Europe, Strasbourg, France, 29 March-2 April 2020.

7. Dezfuli, S.M.; Sabzi, M. Deposition of self-healing thin films by the sol-gel method: A review of layer-deposition mechanisms and activation of self-healing mechanisms. Appl. Phys. A 2019, 125, 557. [CrossRef]

8. Inoue, H.; Iwamoto, T.; Horie, K.; Makishima, A.; Ikemoto, M. Preparation and properties of sol-gel thin films with porphins. J. Opt. Soc. Am. B 1992, 9, 816-818. [CrossRef]

9. Butt, M.A.; Khonina, S.; Kazanskiy, N. Ultrashort inverted tapered silicon ridge-to-slot waveguide coupler at $155 \mu \mathrm{m}$ and 3392 um wavelength. Appl. Opt. 2020, 59, 7821. [CrossRef]

10. Bouajaj, A.; Gonçalves, R.R.; Ferrari, M. Sol-Gel-Derived Erbium-Activated Silica-Titania and Silica-Hafnia Planar Waveguides for $1.5 \mu \mathrm{m}$ Application in C Band of Telecommunication. Spectrosc. Lett. 2014, 47, 381-386. [CrossRef]

11. Reisfeld, R. Sol-Gel processed lasers and related optical materials. In Handbook of Sol-Gel Science and Technology; Klein, L., Aparicio, M., Jitianu, A., Eds.; Springer: Cham, Switzerland, 2016.

12. Karasinski, P.; Tyszkiewicz, C.; Rogozinski, R.; Jaglarz, J.; Mazur, J. Optical rib waveguides based on sol-gel derived silica-titania fims. Thin. Solid Films 2011, 519, 5544-5551. [CrossRef]

13. Khlyustova, A.; Cheng, Y.; Yang, R. Vapor-deposited functional polymer thin films in biological applications. J. Mater. Chem. B 2020, 8, 6588-6609. [CrossRef]

14. Tyszkiewicz, C.; Karasiński, P.; Rogoziński, R. Directional couplers fabricated via a sol-gel route and conventional optical lithography. Opt. Quantum Electron. 2015, 48, 19. [CrossRef]

15. Karasinski, P.; Tyszkiewicz, C.; Rogozinski, R. Rib waveguides based on the sol-gel derived $\mathrm{SiO}_{2}: \mathrm{TiO}_{2}$ films. Photonics Lett. Pol. 2010, 2, 40-42.

16. Chau, Y.-F.C.; Chen, K.-H.; Chiang, H.-P.; Lim, C.M.; Huang, H.J.; Lai, C.-H.; Kumara, N.T.R.N. Fabrication and Characterization of a Metallic-Dielectric Nanorod Array by Nanosphere Lithography for Plasmonic Sensing Application. Nanomaterials 2019, 9 , 1691. [CrossRef]

17. Karasiński, P.; Kaźmierczak, A.; Zięba, M.; Tyszkiewicz, C.; Wojtasik, K.; Kielan, P. Highly Sensitive Sensor Structure Based on Sol-Gel Waveguide Films and Grating Couplers. Electronics 2021, 10, 1389. [CrossRef]

18. Tyszkiewicz, C. Sensing Properties of Four-Layered Planar Waveguides-Theoretical Analysis. Acta Phys. Pol. A 2012, 122, 908-914. [CrossRef]

19. Jaglarz, J.; Dulian, P.; Karasiński, P.; Winkowski, P. Scattering Phenomena in Porous Sol-Gel-Derived Silica Films. Coatings 2020, 10, 509. [CrossRef]

20. Karasiński, P.; Domanowska, A.; Gondek, E.; Sikora, A.; Tyszkiewicz, C.; Skolik, M. Homogeneity of sol-gel derived silica-titania waveguide films-Spectroscopic and AFM studies. Opt. Laser Technol. 2019, 121, 105840. [CrossRef]

21. Weissman, Z. Evanescent field sensors with periodically segmented waveguides. Appl. Opt. 1997, 36, 1218-1222. [CrossRef] [PubMed]

22. Butt, M.A.; Kazanskiy, N.L. Mode sensitivity analysis of vertically arranged double hybrid plasmonic waveguide. Optoelectron. Adv. Mater. Rapid Commun. 2020, 14, 385-388.

23. Odeh, M.; Twayana, K.; Sloyan, K.; Villegas, J.E.; Chandran, S.; Dahlem, M.S. Mode Sensitivity Analysis of Subwavelength Grating Slot Waveguides. IEEE Photon. J. 2019, 11, 1-10. [CrossRef]

24. Butt, M.A.; Khonina, S.N.; Kazanskiy, N.L. Device performance of standard strip, slot and hybrid plasmonic $\mu$-ring resonator: A comparative study. Waves Random Complex Media 2020, 1-10. [CrossRef]

25. Butt, M.A.; Khonina, S.N.; Kazanskiy, N.L. A highly sensitive design of subwavelength grating double-slot waveguide microring resonator. Laser Phys. Lett. 2020, 17, 076201. [CrossRef]

26. Butt, M.A.; Khonina, S.N.; Kazanskiy, N.L. Sensitivity Enhancement of Silicon Strip Waveguide Ring Resonator by Incorporating a Thin Metal Film. IEEE Sens. J. 2019, 20, 1355-1362. [CrossRef]

27. Fu, M.; Zheng, Y.; Li, G.; Hu, H.; Pu, M.; Oxenløwe, L.K.; Frandsen, L.H.; Li, X.; Guan, X. High-Q titanium dioxide micro-ring resonators for integrated nonlinear photonics. Opt. Express 2020, 28, 39084-39092. [CrossRef] [PubMed]

28. Cai, D.-P.; Lu, J.-H.; Chen, C.-C.; Lee, C.-C.; Lin, C.-E.; Yen, T.-J. High Q-factor microring resonator wrapped by the curved waveguide. Sci. Rep. 2015, 5, 10078. [CrossRef]

29. Olyaee, S.; Seifouri, M.; Karami, R.; Mohebzadeh-Bahabady, A. Designing a high sensitivity hexagonal nano-cavity photonic crystal resonator for the purpose of seawater salinity sensing. Opt. Quantum Electron. 2019, 51, 97. [CrossRef]

30. Cheng, X.; Hong, J.; Spring, A.M.; Yokoyama, S. Fabrication of a high-Q factor ring resonator using LSCVD deposited Si_3N_4 film. Opt. Mater. Express 2017, 7, 2182. [CrossRef] 
31. Kazanskiy, N.L.; Khonina, S.N.; Butt, M.A. Subwavelength Grating Double Slot Waveguide Racetrack Ring Resonator for Refractive Index Sensing Application. Sensors 2020, 20, 3416. [CrossRef]

32. Khonina, S.N.; Kazanskiy, N.L.; Butt, M.A. Evanescent Field Ratio Enhancement of a Modified Ridge Waveguide Structure for Methane Gas Sensing Application. IEEE Sens. J. 2020, 20, 8469-8476. [CrossRef]

33. Consani, C.; Dubois, F.; Auböck, G. Figures of merit for mid-IR evanescent-wave absorption sensors and their simulation by FEM methods. Opt. Express 2021, 29, 9723-9736. [CrossRef] [PubMed]

34. Wang, Y.; Gao, B.; Zhang, K.; Yuan, K.; Wan, Y.; Xie, Z.; Xu, X.; Zhang, H.; Song, Q.; Yao, L.; et al. Refractive Index Sensor Based on Leaky Resonant Scattering of Single Semiconductor Nanowire. ACS Photon. 2017, 4, 688-694. [CrossRef]

35. Teng, C.; Yu, F.; Ding, Y.; Zheng, J. Refractive index sensor based on multi-mode plastic optical fiber with long period grating. Proc. SPIE 2017, 10231, 102311M. [CrossRef]

36. Carlborg, C.F.; Gylfason, K.B.; Kaźmierczak, A.; Dortu, F.; Polo, M.J.B.; Catala, A.M.; Kresbach, G.; Sohlström, H.; Moh, T.; Vivien, L.; et al. A packaged optical slot-waveguide ring resonator sensor array for multiplex label-free assays in labs-on-chips. Lab Chip 2010, 10, 281-290. [CrossRef] [PubMed]

37. TalebiFard, S.; Schmidt, S.; Shi, W.; Wu, W.; Jaeger, N.A.F.; Kwok, E.; Ratner, D.M.; Chrostowski, L. Optimized sensitviity of silicon-on-insulator (SOI) strip waveguide resonator sensor. Biomed. Opt. Express 2017, 8, 500-511. [CrossRef] 